\title{
Infecções Sexualmente Transmissíveis no contexto de privação de liberdade
}

\author{
Sexually Transmitted Infections in the context of deprivation of liberty \\ Infecciones de Transmisión Sexual en el contexto de la privación de libertad
}

Recebido: 17/01/2021 | Revisado: 19/01/2021 | Aceito: 21/01/2021 | Publicado: 25/01/2021

Nádia Vicência do Nascimento Martins
ORCID: https://orcid.org/0000-0002-8166-644X
Universidade do Estado do Pará, Brasil
E-mail: nadia.martins@uepa.br
Lucia Yasuko Izumi Nichiata
Universidade de São Paulo, Brasil
E-mail: izumi@usp.br
Maria Rita Bertolozzi
ORCID: https://orcidor/0000-0001-6515-4404
Unido
UnCID: https://orcid.org/0000-0002-5009-5285
Universidade de São Paulo, Brasil
E-mail: mrbertol@usp.br
Adriana Vanessa Ribeiro Mafra
ORCID: https://orcid.org/0000-0001-6014-7715
Universidade do Estado do Pará, Brasil
E-mail: mafra.dry @ gmail.com
Randerson José de Araujo Sousa
ORCID: https://orcid.org/0000-0003-3723-8955
Universidade do Estado do Pará, Brasil
E-mail: rajoarso@ gmail.com

\section{Resumo}

Identificar casos auto referidos e confirmados de IST em mulheres privadas de liberdade. Trata-se de estudo de caso, exploratório, descritivo, com corte transversal, com abordagem quantitativa, realizado com 62 mulheres do Complexo Prisional de Santarém, Pará. Os resultados evidenciaram que 56,5\% das mulheres tinham baixa escolaridade, 91,9\% se autodeclararam pardas e negras, 59,7\% encontravam-se na faixa etária de 21 a 40 anos, 59,7\% informaram que não usavam preservativo. A prevalência de IST auto referida foi de $25,8 \%$ (sífilis e gonorreia), porém, foram identificados e confirmados $19,4 \%$ casos de sífilis e 7,0\% de casos HPV, não foram identificadas outras IST no estudo. Constatouse o quão distante o sistema prisional brasileiro está do ideal humanitário, além da necessidade de reconhecer as mulheres em privação de liberdade como capazes de tomarem decisões sobre seus corpos e sua saúde, neste sentido, é fundamental que estas mulheres, tenham condições dignas e oportunidades de promoção e educação em saúde acerca das IST, bem como atendimento dentro dos princípios garantidos pelas políticas públicas, de acessibilidade, integralidade, resolutividade e humanização da assistência em saúde.

Palavras-chave: Enfermagem; Infecções sexualmente transmissíveis; População privada de liberdade; Saúde da mulher.

\begin{abstract}
Identify self-reported and confirmed cases of STI in women deprived of their liberty. This is an exploratory, descriptive, cross-sectional case study with a quantitative approach, performed with 62 women from the Prison Complex of Santarém, Pará. The results showed that 56,5\% of the women had low schooling, 91,9\% declared themselves to be black and brown-skinned, 59,7\% were in the age group of 21 to 40 years, 59,7\% said they did not use a condom. The prevalence of self-reported STI was 25,8\% (syphilis and gonorrhea), but 19,4\% of syphilis cases and $7.0 \%$ of HPV cases were identified and confirmed, no other STI were identified in the study. It was noted how distant the Brazilian prison system is from the humanitarian ideal, besides the need to recognize women in deprivation of liberty as capable of making decisions about their bodies and their health, in this sense, it is fundamental that these women, have dignified conditions and opportunities for health promotion and education about STI, as well as services within the principles guaranteed by public policies, of accessibility, completeness, resolution and humanization of health care.
\end{abstract}

Keywords: Nursing; Sexually transmitted infections; Population deprived of liberty; Women's health.

\section{Resumen}

Identificar los casos autodeclarados y confirmados de ITS en mujeres privadas de libertad. Se trata de un estudio de caso exploratório, descriptivo y transversal, con un enfoque cuantitativo, realizado con 62 mujeres del Complejo Penitenciario de Santarém, Pará. Los resultados mostraron que el 56,5\% de las mujeres tenían un bajo nivel de escolaridad, el 91,9\% se declararon morenas y negras, el 59,7\% estaban en el grupo de edad de 21 a 40 años, el 59,7\% 
informaron que no usaban condones. La prevalencia de las ITS autoinformadas fue del 25,8\% (sífilis y gonorrea), sin embargo, se identificaron y confirmaron el 19,4\% de los casos de sífilis y el 7,0\% de los casos de VPH, y no se identificó ninguna otra ITS en el estudio. Se verificó cuán lejos está el sistema penitenciario brasileño del ideal humanitario, además de la necesidad de reconocer a las mujeres en privación de libertad como capaces de tomar decisiones sobre su cuerpo y su salud, en este sentido, es fundamental que estas mujeres, tengan condiciones dignas y oportunidades de promoción de la salud y educación sobre las ITS, así como la atención dentro de los principios garantizados por las políticas públicas, accesibilidad, integridad, resolución y humanización de la atención en salud.

Palabras clave: Enfermería; Infecciones de transmisión sexual; Población privada de libertad; Salud de la mujer.

\section{Introdução}

Em diferentes países, as Infecções Sexualmente Transmissíveis (IST) historicamente antigas permanecem presentes, como a sífilis, a gonorreia, a donovanose, o herpes genital e o Papilomavírus Humano (HPV), somadas na década de 1980, ao surgimento da epidemia da aids e ao reconhecimento da importância da hepatite B como IST (Naud, 1993; Buchalla \& Paiva, 2002; Dallabetta et al., 1997; Reis \& Bernardes, 2011).

A Organização Mundial de Saúde (2013) estimou cerca de 500 milhões de casos novos de IST curáveis, acometendo homens e mulheres entre 15 e 49 anos, desses, cerca de 10 a 12 milhões de casos ocorrem no Brasil. As IST estão entre um dos problemas de saúde mais comuns em todo o mundo e ainda se desconhece sua magnitude, dado que, do conjunto de infecções, poucas são informadas de forma sistematizada e; quando estão minimamente organizados os dados, estes se referem somente à sífilis e à aids que são as únicas doenças contabilizadas estatisticamente, por serem de notificação compulsória (WHO, 2013; Brasil, 2015).

Apesar da falta de informações mais precisas sobre a extensão das IST, no Brasil, considera-se alta sua incidência e prevalência e com consequências que extrapolam a intimidade da vida sexual privada, com repercussões biológicas e psicossociais tanto do ponto de vista individual, como do coletivo, configurando-se um grave problema de Saúde Pública (Brasil, 2015; Belda et al., 2009).

Em 2017, a OMS estimou que mais de 1 milhão de novos casos de IST eram adquiridas diariamente em todo o mundo. As ITS como um problema de Saúde Pública global, tem seu manejo muitas vezes comprometido pela diversidade de agentes patogênicos, pelo estigma social e por sinais e sintomas comumente leves ou mesmo inexistentes (OMS, 2017).

As IST, mesmo que, por vezes, de forma mais discreta, apresentam sequelas proeminentes como em casos de mulheres grávidas infectadas com sífilis, em que cerca de um terço tem resultados adversos durante o parto, incluindo natimortos; ou em casos de infecção por HPV com registros de cerca de 266 mil mortes por câncer cervical por ano, além de possibilidade de apresentar como sequela a Doença Inflamatória Pélvica (DIP); destacando-se, ainda, a infertilidade feminina; o parto prematuro e recém-nascidos com baixo peso ao nascer (OMS, 2016).

Estudos realizados nos últimos 30 anos mostram que, o advento da epidemia da aids, trouxe consequências devastadoras para a população em geral, configurando-se como um dos maiores desafios para a Saúde Coletiva. Estima-se que mais de 7.000 pessoas são infectadas com o HIV diariamente, e que uma (1) pessoa morre a cada 20 segundos por uma doença relacionada à aids (doenças oportunistas). A aids é considerada a $5^{\mathrm{a}}$ causa de morte entre adultos e a principal causa de morte entre as mulheres com idade entre 15 e 49 anos (Unaids, 2013; Martins et al., 2014; Mome et al., 2018).

Destaca-se que a presença de IST aumenta a susceptibilidade ao HIV. Há várias intervenções para a prevenção, e, embora o preservativo masculino seja eficaz na redução de sua transmissão das IST, a negociação, em relação ao uso do preservativo masculino com parceiros em muitos países, para as mulheres, especialmente na África Subsaariana é muito difícil, sendo apontada como um elemento que agrava a situação de vulnerabilidade das mulheres ao adoecimento (Mome et al., 2018).

As conhecidas "populações-chave" são segmentos populacionais que merecem atenção especial, pois podem apresentar fragilidades que aprofundam as vulnerabilidades ao HIV/aids. Estas incluem: homens que fazem sexo com homens, 
pessoas que usam álcool e outras drogas, pessoas privadas de liberdade, trabalhadores do sexo e transexuais destaca-se que mulheres autodeclaradas como profissionais do sexo têm até 12 vezes mais chances de contrair o HIV quando comparadas com mulheres em geral. As populações-chave podem estar frequentemente em contextos de vulnerabilidade individual e social que podem remeter à transmissão do HIV e demais IST. É necessário identificar, avaliar e diminuir, se houver, as possíveis barreiras de acesso das populações chaves aos programas de testagem e tratamento, devido ao estigma e discriminação (Boni et al., 2014; OPAS, 2017).

Além de causar impacto na saúde individual, a epidemia de HIV/aids e outras IST, funciona como uma lente de aumento, para a visualização dos males da sociedade e das deficiências dos sistemas sociais. Desta forma, a implantação e a implementação de políticas públicas voltadas a respostas à epidemia podem proporcionar o fortalecimento dos sistemas sociais, como a justiça social e reforçar a prestação de serviços considerados essenciais aos segmentos mais vulneráveis da sociedade.

Destaca-se a necessidade de se obter equilíbrio entre a intensificação de respostas as políticas públicas em países com as maiores taxas de incidência e prevalência, assim como a identificação de contextos de vulnerabilidades em outros contextos como: em grandes centros urbanos, áreas de aglomeração, em que o impacto do HIV afeta comunidades específicas à exemplo de, homens que fazem sexo com homens, profissionais do sexo, pessoas usuárias de drogas e, ainda, indivíduos em contexto de privação de liberdade (UNAIDS, 2013; UNAIDS, 2015).

Desta forma, e levando em consideração que o número de mulheres reclusas vem aumentando significativamente no Brasil e que o ambiente prisional é uma condição que, de partida expressa um contexto de vulnerabilidade, destaca-se como fundamental a realização de estudos que possam abordar a temática "Saúde da Mulher no sistema prisional. Nesta perspectiva, objetivou-se identificar casos auto referidos e confirmados de Infecções Sexualmente Transmissíveis em mulheres privadas de liberdade em Santarém, Pará, Brasil.

\section{Metodologia}

Trata-se de pesquisa exploratória, descritiva, transversal, com abordagem quantitativa que segundo Pereira (2018) a coleta de dados quantitativos ou numéricos é realizada por meio do uso de medições de grandezas, de técnicas matemáticas como é o caso das porcentagens, estatísticas e probabilidades, métodos numéricos, métodos analíticos e geração de equações e/ou fórmulas matemáticas aplicáveis a algum processo.

A pesquisa foi realizada no Centro de Recuperação Agrícola Silvio Hall de Moura (CRASHM) com sede no município de Santarém, Estado do Pará. O CRASHM é um Centro de Recuperação misto, ou seja, tem sob sua custódia homens e mulheres, em regime de reclusão, fechado, semiaberto ou provisório.

Foram convidadas a participar do estudo mulheres privadas de liberdade, em qualquer idade e que, no período da coleta de dados cumpriam pena de reclusão no CRASHM.

Foram critérios de exclusão mulheres privadas de liberdade que declarassem qualquer dificuldade cognitiva, visual ou auditiva no momento da aplicação do questionário e aquelas que por determinação judicial estivessem impedidas da participação em quaisquer atividades, sejam por medidas restritivas e ou disciplinares. No entanto, destaca-se que não houve situação de exclusão durante o estudo.

O período de coleta de dados foi de setembro de 2016 a fevereiro de 2017, neste intervalo foram aplicados 62 questionários, o que representou a totalidade da população feminina privada de liberdade em Santarém, Pará.

Foi utilizado um instrumento para a captação dos dados, composto por variáveis referentes ao perfil das participantes do estudo e conhecimento acerca das IST, e a organização dos serviços de saúde no Sistema Penitenciário de Santarém, Pará. 
O instrumento foi estruturado com respostas fechadas, buscando caracterizar as participantes em termos socioeconômicos e demográficos, sobre conhecimento a respeito de IST, antecedentes ginecológicos, além de práticas sexuais e preventivas da mulher privada de liberdade.

Após a apresentação da proposta de pesquisa à Superintendência do Sistema Penitenciário (SUSIPE) e ao Centro de Recuperação Agrícola Silvio Hall de Moura (CRASHM), foi realizada visita à Unidade Prisional (UP), com o intuito de apresentar a proposta de pesquisa ao corpo administrativo e assistencial.

A partir da sensibilização da equipe multiprofissional sobre a temática, assim como a exposição dos objetivos e etapas do projeto de pesquisa, o cronograma de visitas ao CRASHM foi definido, com dias e horários determinados pela direção e equipe de segurança do sistema penal.

A primeira visita à ala feminina do CRASHM para a sensibilização dos potenciais participantes do estudo, foi realizada acompanhada pela coordenação da equipe de saúde e de agentes prisionais.

Nessa ocasião foi apresentado o projeto, seus objetivos, as etapas a serem realizadas e o convite formal para participação, tendo sido apresentado o Termo de Consentimento Livre e Esclarecido (TCLE).

1 Etapa: Caracterização do perfil socioeconômico e demográfico, identificação de conhecimento e práticas preventivas das IST.

Antecedendo a apresentação desta etapa, vale destacar as dificuldades encontradas, e que estas dizem respeito ao quantitativo insuficiente de agentes prisionais femininos, para realizar a condução e escolta das mulheres até a Unidade Básica de Saúde Prisional (UBSp), durante todo o processo de aplicação do questionário.

Aplicou-se o questionário, face a face com a participante, com duração média de 30 minutos, no consultório de enfermagem da UBSp. As mulheres eram conduzidas algemadas e escoltadas por uma agente prisional feminino, a depender de seu histórico e comportamento, as algemas eram retiradas, e a agente prisional permaneceu externamente à sala, o que garantiu à privacidade das mulheres e o sigilo das informações prestadas, evitando constrangimento no momento das respostas.

$2^{\text {a }}$ Etapa: Estimativa de Prevalência auto referida de IST, identificação de casos suspeitos e confirmados de sífilis, HIV, hepatite B e C e HPV

A Unidade de Referência Especializada em Saúde (URES) é um serviço de referência, para o atendimento de casos de média complexidade, oriundos das Unidades Básicas de Saúde de Santarém, assim como de outros municípios da região, foi a unidade de saúde responsável pela realização dos exames e, emissão dos laudos. A equipe multiprofissional da URES compareceu à UBSp para realizar o rastreamento por meio de Teste Rápido (TR) de HIV, sífilis, hepatite B (HBV) e hepatite C (HCV) e, em casos de TR reagente para sífilis, foram solicitados o exame de VDRL quantitativo, para a determinação do estado sorológico.

Participaram desta etapa 61 mulheres, com registro de 01 recusa em realizar os testes, após algumas tentativas de oferta. Os TR foram realizados na ala feminina do CRASHM, em uma área coberta, e adaptada para garantia de privacidade no aconselhamento pré-teste, no entanto, mesmo tendo o resultado em 30 minutos, estes foram entregues posteriormente, durante as consultas médica e de enfermagem.

Além dos TR, às mulheres foi ofertada a realização de exames citopatológicos (Preventivo do Câncer de Colo Uterino-PCCU). Os exames preventivos foram realizados em sala adequada e reservada (consultório ginecológico da UBSp), em dias e horários previamente agendados, 55 exames preventivos foram coletados pela pesquisadora e 01 profissional Enfermeira, as informações foram registradas em formulário próprio do Programa Preventivo do Câncer de Colo Uterino. Após a coleta e fixação em lâmina, as amostras biológicas foram enviadas à URES para análise e posterior liberação dos laudos com respectivos resultados. 
A partir da liberação dos laudos dos TR e PCCU, foram agendadas consultas médicas e de enfermagem para a entrega dos respectivos resultados as participantes do estudo.

Os resultados não reagentes às IST e os resultados negativos para inflamação pelo PCCU, foram entregues durante as consultas e, na oportunidade, as mulheres foram orientadas quanto ao resultado, aos cuidados quanto à manutenção de medidas preventivas à saúde, uso de preservativos e realização de exames preventivos periodicamente.

Os resultados reagentes para IST, identificados por TR e os resultados sugestivos de inflamação e ou infecção por microrganismos identificados no PCCU, foram entregues durante as consultas e, na oportunidade, as mulheres foram orientadas quanto ao tratamento específico, quanto à importância da adesão ao tratamento, e foram encaminhadas para avaliação psicológica e da assistência social.

Os casos sugestivos de HPV foram encaminhados para reavaliação, por uma equipe médica especializada. Para tanto, solicitou-se o agendamento de consultas especializadas, via central de regulação de consultas, para a URES ou Centro de Referência em Saúde da Mulher (CRS Mulher), para a continuidade do tratamento específico.

Os dados obtidos no questionário sobre o perfil socioeconômico e demográfico das mulheres, e de conhecimentos e práticas sobre prevenção das IST, foram tabulados em planilha eletrônica do programa Microsoft Excel 2013, processados e analisados com o auxílio do programa computacional para análise estatística "Statistical Package for the Social Sciences (SPSS), versão 21.0".

Para a execução desta pesquisa foram obedecidas as normas éticas descritas na Resolução 466/2012 do Conselho Nacional de Saúde (CNS), tendo sido aprovado pelo Comitê de Ética da Escola de Enfermagem da Universidade de São Paulo (CAAE nº 57138016.5.0000.5392 e Parecer de aprovação: no 1.725.469).

\section{Resultados}

Responderam ao questionário, 62 mulheres. A Tabela 1, mostra (47:75,8\%) autodeclararam-se pardas, (35:56,5\%) concluíram o ensino fundamental, e (37:59,7\%) encontravam-se na faixa etária de 21 a 40 anos. 
Tabela 1 - Perfil socioeconômico e demográfico das mulheres privadas de liberdade. Santarém, Pará, 2017.

\begin{tabular}{|c|c|c|c|}
\hline Perfil sociodemográfico & FREQUÊNCIA & $(\%)$ & $X^{2}$ \\
\hline $\operatorname{RAÇA}(n=62)$ & & & $\mathrm{p}=<0,001$ \\
\hline Branca & 5 & 8,1 & \\
\hline Negra & 10 & 16,1 & \\
\hline Parda & 47 & 75,8 & \\
\hline ESCOLARIDADE $(n=62)$ & & & $\mathrm{p}=<\mathbf{0 , 0 0 1}$ \\
\hline Nenhuma & 1 & 1,6 & \\
\hline Ensino Fundamental completo & 35 & 56,5 & \\
\hline Ensino Médio completo & 25 & 40,3 & \\
\hline Ensino Superior incompleto & 1 & 1,6 & \\
\hline FAIXA ETÁRIA (n=62) & & & $p=0,003$ \\
\hline $18 \dashv 20$ anos & 14 & 22,6 & \\
\hline $21 \dashv 30$ anos & 22 & 35,5 & \\
\hline $31-40$ anos & 15 & 24,2 & \\
\hline $41 \dashv 50$ anos & 6 & 9,6 & \\
\hline $51 \dashv 60$ anos & 5 & 8,1 & \\
\hline
\end{tabular}

* Teste $X^{2-}$ Qui quadrado; $\mathrm{p}=$ valor de " $\mathrm{p}$ ".

Fonte: Autores (2018).

Na análise estatística dos dados apresentados na Tabela 1, por meio do teste Qui quadrado $\left(X^{2}\right)$, as variáveis socioeconômicas e demográficas demonstram a significância estatística $(p \leq 0,05)$, com exceção da variável faixa etária. 
Tabela 2 - Contextos de práticas sexuais de mulheres privadas de liberdade. Santarém, Pará, 2017.

\begin{tabular}{|c|c|c|c|}
\hline PRÁTICAS SEXUAIS & FREQUÊNCIA & $(\%)$ & $X^{2}$ \\
\hline Usa preservativo com parceiro fixo $(n=62)$ & & & $\mathrm{p}=<0,001$ \\
\hline Não & 37 & 59,7 & \\
\hline Sim & 24 & 38,7 & \\
\hline Não informado & 1 & 1,6 & \\
\hline Se não, qual o motivo? (n=37) & & & $\mathrm{p}=\mathbf{0 , 1 0 7}$ \\
\hline Não gosta & 14 & 22,6 & \\
\hline Não consegue negociar com o parceiro & 25 & 40,3 & \\
\hline Outro motivo não informado & 27 & 43,5 & \\
\hline Tem parceiro sexual fixo? $n=(62)$ & & & $\mathrm{p}=\mathbf{0 , 0 5 6}$ \\
\hline Não & 23 & 37,1 & \\
\hline Sim & 39 & 62,9 & \\
\hline Realização de Preventivo do Câncer de Col & PCCU)? & $\mathrm{p}=<0,001$ & \\
\hline Sim & 46 & 74,2 & \\
\hline Não & 16 & 25,8 & \\
\hline Se sim. Em que intervalo de tempo? & & & $\mathbf{p}=\mathbf{0 , 0 1 7}$ \\
\hline 2008 a 2010 & 3 & 4,8 & \\
\hline 2011 a 2013 & 5 & 7,0 & \\
\hline 2014 & 12 & 19,4 & \\
\hline 2015 & 10 & 16,1 & \\
\hline 2016 & 16 & 25,8 & \\
\hline
\end{tabular}

* Teste $X^{2-}$ Qui quadrado; $\mathrm{p}=$ valor de " $p$ ".

Fonte: Autores (2018).

Quanto ao contexto de práticas sexuais das mulheres (Tabela 2), (37:59,7\%) informaram não usar preservativo, $(25: 40,3 \%)$ indicaram como principal "motivo" o fato de não conseguir negociar com o parceiro. A maioria das mulheres, (39:62,9\%), informou ter parceiro fixo.

Sobre o PCCU, (46:74,2\%) informaram ter realizado o exame pelo menos uma vez na vida e, para $(16: 25,8 \%)$ esta, foi a primeira vez que realizou o PCCU. A análise estatística das variáveis que representam os contextos de práticas sexuais mostrou diferença estatística significativa entre as opções. No entanto, as variáveis motivo de não usar preservativo e não ter parceiro sexual fixo, não apresentaram diferença estatística. 
Tabela 3 - Distribuição de mulheres segundo Infecções Sexualmente Transmissíveis auto referidas, suspeitas e confirmadas por meio de diagnóstico laboratorial. Santarém, Pará, 2017.

\begin{tabular}{|c|c|c|c|}
\hline IST & FREQUENCIA & $(\%)$ & $X^{2}$ \\
\hline \multicolumn{4}{|c|}{ Já teve doença transmitida através das relações sexuais (doença venérea, doença de rua)? (n=62) } \\
\hline Não & 44 & 71,0 & $\mathrm{p}=<\mathbf{0 , 0 0 1}$ \\
\hline Sim & 16 & 25,8 & \\
\hline Não informado & 02 & 3,2 & \\
\hline \multicolumn{4}{|c|}{ Qual doença transmitida através das relações sexuais já teve? } \\
\hline Sífilis & 12 & 19,4 & $\mathrm{p}=<\mathbf{0 , 0 0 1}$ \\
\hline Gonorreia & 02 & 3,2 & \\
\hline Não sabe informar & 02 & 3,2 & \\
\hline Não informado & 02 & 3,2 & \\
\hline Não teve & 44 & 71,0 & \\
\hline \multicolumn{4}{|c|}{ Infecções Sexualmente Transmissíveis confirmadas por meio de teste rápido. } \\
\hline \multicolumn{4}{|l|}{ SÍFILIS } \\
\hline Reagente & 14 & 22,6 & $\mathrm{p}=<\mathbf{0 , 0 0 1}$ \\
\hline Não reagente & 47 & 75,8 & \\
\hline Recusa em realiza & 01 & 1,6 & \\
\hline \multicolumn{4}{|c|}{ VDRL quantitativo realizado (exame confirmatório de doença ativa) $\mathbf{n}=(\mathbf{1 4})$. } \\
\hline Reagente & 04 & 28,6 & \\
\hline \multicolumn{4}{|c|}{ Exame citopatológico $-\mathbf{P C C U}-$ Resultados sugestivos $-\mathbf{n}=(\mathbf{5 7})$} \\
\hline$H P V$ & 04 & 7,02 & $p=0,001$ \\
\hline Lactobacillus sp & 09 & 15,79 & \\
\hline Bacilos (Gardnerella) & 21 & 36,84 & \\
\hline Flora Mista & 19 & 33,33 & \\
\hline Cocos, Bacilos & 04 & 7,02 & \\
\hline
\end{tabular}

* Teste $X^{2-}$ Qui quadrado; $\mathrm{p}=$ valor de " $\mathrm{p}$ ".

Fonte: Autores (2018).

Quanto a distribuição das IST auto referidas e confirmadas por meio de diagnóstico laboratorial, obteve-se diferença estatisticamente significativa entre as opções de resposta de cada variável, dezesseis $(25,8 \%)$ mulheres informaram terem tido alguma IST, e (12:19,4\%) indicaram a sífilis (Tabela 3).

Neste estudo, não houve resultados reagentes para HIV, hepatites virais B e C, porém, (14:22,6\%) tiveram resultado reagente ao Teste Rápido (TR) para sífilis. Estas mulheres realizaram o VDRL quantitativo para identificar se a infecção estava ativa ou se se tratava de cicatriz sorológica. Quatro $(28,6 \%)$ mulheres apresentaram-se reagentes, e tratadas conforme protocolo do Ministério da Saúde para o tratamento de sífilis. 
Das 55 mulheres que aceitaram realizar o PCCU, (21:36,8\%) tiveram resultado sugestivo para Gardnerella vaginalis e (4:7,0\%) apresentaram resultado sugestivo para HPV. Estas foram encaminhadas para o Serviço de Referência de Média Complexidade do município de Santarém, para consulta médica especializada e realização do exame de colposcopia para a confirmação do resultado e tratamento específico.

Ao analisar as características sociodemográficas evidencia-se que, na população investigada com resultado reagente, a maior concentração encontrava-se entre adultos jovens (6:42,9\%) na faixa etária entre 25 a 34 anos de idade.

Tabela 4 - Distribuição das participantes do estudo, segundo faixa etária, número de parceiros e histórico de Infecções Sexualmente Transmissíveis por resultado de teste sorológico de Sífilis. Santarém, Pará, 2017.

\begin{tabular}{|c|c|c|c|c|}
\hline \multirow{2}{*}{ VARIÁVEL } & \multicolumn{2}{|c|}{ REAGENTE } & \multicolumn{2}{|c|}{ NÃO REAGENTE } \\
\hline & $\mathrm{n}=14$ & $\%(100)$ & $\mathrm{n}=47$ & $\%(100)$ \\
\hline \multicolumn{5}{|l|}{ FAIXA ETÁRIA } \\
\hline $18 \dashv 24$ anos & 03 & 21,4 & 24 & 51,1 \\
\hline $25 \dashv 34$ anos & 06 & 42,9 & 08 & 17,0 \\
\hline $35 \dashv 44$ anos & 01 & 7,1 & 12 & 25,5 \\
\hline Mais de 44 anos & 04 & 28,6 & 03 & 6,4 \\
\hline \multicolumn{5}{|l|}{ NÚMERO DE PARCEIROS } \\
\hline Até 03 parceiros sexuais & 02 & 14,2 & 14 & 29,7 \\
\hline De 04 a 09 parceiros sexuais & 02 & 14,2 & 12 & 25,5 \\
\hline Mais de 09 parceiros sexuais & 02 & 14,2 & 17 & 36,1 \\
\hline Vários parceiros sexuais & 08 & 57,1 & 04 & 8,51 \\
\hline \multicolumn{5}{|l|}{ HISTÓRICO DE IST } \\
\hline Sim & 10 & 71,4 & 06 & 12,8 \\
\hline Não & 03 & 21,4 & 40 & 85,1 \\
\hline Não informado & 01 & 7,2 & 01 & 2,1 \\
\hline
\end{tabular}

Fonte: Autores (2018).

Quanto ao número de parceiros sexuais, a maioria declarou ter mantido relações sexuais com "vários" parceiros (8:57,1\%), duas (2) mulheres 14,2\% declararam: manter relação sexual com "mais de 9 parceiros sexuais"; e o mesmo montante entre " 4 a 9 parceiros" e com "até 3 parceiros" respectivamente (Tabela 4).

Evidencia-se que a população investigada com resultado não reagente para sífilis, em relação às características sociodemográficas é predominantemente jovem (24:51,1\%) estando na faixa etária entre 18 a 24 anos de idade.

Das mulheres com resultados não reagente, $(4: 8,5 \%)$ declaram ter se envolvido com "vários" parceiros sexuais, $(17: 36,1 \%)$ responderam ter se envolvido com "mais de 9 parceiros", e (12:25,5\%) relataram envolvimento com "de 4 a 9 parceiros sexuais", enquanto que (14:29,7\%) declararam envolvimento "com até 3 parceiros sexuais".

Ao se analisar a história prévia de sífilis, obtida por meio da aplicação do questionário, nota-se que de 14 mulheres com resultado reagente no teste rápido de sífilis, $(12: 85,0 \%)$ relataram histórico de sífilis anterior e $(2: 15,0 \%)$ foram casos 
novos. Esses dados evidenciam a vulnerabilidade a que esse grupo de mulheres é exposto, assim como a dificuldade de acesso aos serviços de saúde no que concerne ao tratamento, podendo justificar a alta prevalência da infecção pelo T. pallidum na população privada de liberdade.

Tabela 5 - Distribuição das participantes do estudo, segundo faixa etária e alterações nos resultados de Exames Citopatológicos de Colo Uterino. Santarém, Pará, 2017.

\section{Alterações Citopatológicas}

\begin{tabular}{|c|c|c|c|}
\hline & Inflamação & $\begin{array}{c}\text { Lesão Intraepitelial de } \\
\text { Baixo Grau }\end{array}$ & Lesão Intraepitelial de Alto Grau \\
\hline Faixa etária (anos) & $\mathrm{n}=51(\%)$ & $\mathrm{n}=03(\%)$ & $\mathrm{n}=01(\%)$ \\
\hline $18 \dashv 25$ & $26(51,0)$ & $2(66,7)$ & - \\
\hline $26 \dashv 30$ & $7(13,7)$ & - & $1(100)$ \\
\hline $31 \dashv 35$ & $4(7,8)$ & - & - \\
\hline $36 \dashv 40$ & $7(13,7)$ & $1(33,3)$ & - \\
\hline $41 \dashv 45$ & $1(2,0)$ & - & - \\
\hline $46 \dashv 50$ & $2(3,9)$ & - & - \\
\hline$>51$ & $4(7,8)$ & - & - \\
\hline \multicolumn{4}{|c|}{ Intervalo de tempo na realização do Preventivo do Câncer de Colo Uterino (anos) } \\
\hline Anualmente & $13(25,5)$ & $1(33,3)$ & - \\
\hline 2 & $7(13,7)$ & - & $1(100)$ \\
\hline 3 & $9(17,6)$ & $2(66,7)$ & - \\
\hline 5 & $3(5,9)$ & - & - \\
\hline$>5$ & $3(5,9)$ & - & - \\
\hline Nunca tinha realizado & $16(31,4)$ & - & - \\
\hline
\end{tabular}

Fonte: Autores (2018).

Na Tabela 5 estão distribuídos segundo faixa etária e intervalo de realização do PCCU, as alterações encontradas nos Exames Citopatológicos de Colo Uterino. Em relação à faixa etária a maior concentração de mulheres (26:51,0\%) encontravase entre 18 e 25 anos de idade. A maioria (39:68,6\%), já havia realizado o PCCU anteriormente a este estudo, sendo que (29:56,8\%) com intervalo de até três anos; com predominância de (51:92,7\%) resultados conclusivos para "inflamação" (Tabela 5).

Em menor número de mulheres, identificou-se a presença de (3:5,5\%) casos de Lesão Intraepitelial de Baixo Grau e $(1: 1,8 \%)$ caso de Lesão Intraepitelial de Alto Grau, na faixa etária jovem e adulta até 40 anos de idade. Destaca-se a importância da detecção precoce a partir da ampliação da oferta e acesso aos exames preventivos de colo uterino.

Em relação aos principais agentes microbianos encontrados nos exames citopatológicos das 55 mulheres, foram identificados os seguintes resultados: Gardnerella (22:40,0\%), Flora mista (19:34,5\%), Lactobacillus (9:16,4\%), Cândida sp. $(4: 7,3 \%)$ e cocos e outros bacilos $(1: 1,8 \%)$. 
Dezesseis $(25,8 \%)$ mulheres estavam realizando o PCCU pela primeira vez, desta forma, destaca-se que (8:50,0\%) delas declararam ter tido um único parceiro sexual nos últimos seis meses, (9:56,2\%) eram heterossexuais, (10:62,5\%) relataram não fazer uso do preservativo nas relações sexuais, justificado pelo motivo de "não gostar" ou "não consegue negociar com o parceiro".

\section{Discussão}

A vulnerabilidade, em sua dimensão individual, diz respeito às ações relacionadas à prevenção diante de uma situação onde há risco de adoecimento. Envolve aspectos relacionados às características pessoais, emocionais, percepção do risco e atitudes voltadas à adoção de medidas de autoproteção e atitudes em relação à sexualidade, bem como de conhecimentos adquiridos ao longo da vida acerca das IST (Ayres et al., 2003).

Embora, potencialmente, todas as mulheres sejam vulneráveis às IST, há alguns grupos de mulheres particularmente mais expostos à determinadas condições que as tornam mais vulneráveis e, consequentemente, com maiores chances de desenvolver tais infecções.

A raça/etnia é um marcador de vulnerabilidade das mulheres do estudo, com 91,9\% das mulheres pardas e negras, percentual próximo aos presídios femininos do Estado do Pará $(89,0 \%)$ e maior que o percentual encontrado em outros presídios brasileiros, de 62,0\% (Brasil, 2018). É preciso lembrar que, de certa maneira este resultado reflete o perfil geral da população do Estado do Pará e do município de Santarém, onde juntos, pardos e negros, representam 76,7\% e 77,7\%, respectivamente (IBGE, 2015).

Mais da metade das mulheres pesquisadas (59,7\%) eram jovens, entre 21 a 40 anos, na faixa etária caracterizada como período produtivo e reprodutivo da vida da mulher. Comparando este resultado a outras pesquisas realizadas em presídios femininos, com mulheres em faixa etária mais alargada, de 18 a 45 anos, os resultados mostram percentuais de: 88,0\% (Zaninelli, 2015) no Estado do Paraná, 98\% no Estado do Pará e 89,0\% nos presídios brasileiros (Brasil, 2018).

Quanto ao contexto de práticas sexuais das mulheres privadas de liberdade em Santarém, Pará, destaca-se que (37:59,7\%) informaram não usar preservativo nas relações sexuais, ressaltando que esta escolha se fez por vários motivos como: não gostar de usar, pelo desejo de ter filhos, por confiar no parceiro; porém, a maioria concentra-se em "não conseguir negociar o uso do preservativo com o parceiro" motivo indicado por $(25: 40,3 \%)$ mulheres.

Comparando esses achados a outros estudos, onde os resultados estão em conformidade com a atual pesquisa, destacando-se que $(78,7 \%)$ das mulheres encarceradas não usam preservativos nas relações sexuais, sendo que $(21,3 \%)$ informaram usar preservativo, $20 \%$ disseram usar o preservativo masculino e apenas $1,3 \%$ disseram usar o preservativo feminino (Nicolau et al., 2012).

Entretanto, há outros estudos em que 43,6\% das mulheres privadas de liberdade informaram nunca ter usado preservativo e 44,9\% o usavam, porém, de forma eventual (Neri et al., 2011), corroborando os resultados deste estudo em que a maioria (52:83,9\%), informou ter relações sexuais com diferentes pessoas com uso eventual de preservativo, e (24:38,7\%) relatou envolvimento com dois (2) parceiros sexuais nos últimos 6 meses. Destaca-se, ainda, estudos em que a maioria das mulheres privadas de liberdade, informou não fazer uso de preservativo, seja masculino ou feminino (Barbosa et al., 2017).

A maioria das mulheres do presente estudo (39:62,9\%), informaram ter parceiro fixo, destacando-se que o motivo de não usar preservativo está associado à relação de confiança no parceiro, pois viviam relação de compromisso e se sentiam mais protegidas. Desta forma, se pode inferir que estas mulheres ficam impedidas de avaliar os riscos, a exposição e, consequentemente a vulnerabilidade às IST (Nicolau et al., 2012). Além do mais o fato de não usar preservativo ou usá-lo eventualmente evidencia a vulnerabilidade de mulheres às IST (Neri et al., 2011), assim como a gravidez indesejada. Desta 
forma, vale ressaltar a necessidade de implantação e implementação de atividades de educação em saúde, voltadas à promoção, prevenção e proteção da saúde da mulher, seja dentro ou fora das penitenciárias.

Em contraponto, um estudo com mulheres em liberdade, revela que apenas 50\% das participantes referiram utilizar métodos contraceptivos eficazes na prevenção de gravidez, o que, não as exime da infecção por qualquer IST, mantendo a vulnerabilidade ao adoecimento (Rufino et al., 2016). A construção do saber em saúde e o uso de preservativo em todas as relações sexuais, constitui uma alternativa eficaz na prevenção das IST/HIV/aids. Porém, pelo fato de envolver inúmeros aspectos sociais, culturais e pessoais, a negociação com o parceiro ainda é restrita, pois ainda que as mulheres reconheçam sua vulnerabilidade, muitas sabem que o poder de reduzir o risco e decidir o uso do preservativo, é limitado a seu parceiro.

Quanto aos cuidados de saúde da mulher, como a realização do PCCU, a pesquisa mostra um percentual de mulheres que já haviam realizado o exame pelo menos uma vez na vida $(74,2 \%)$, mostrando que, de alguma forma, estas mulheres têm tido acesso ao exame, no sistema prisional, ou fora dele. Todavia, destaca-se que o exame preventivo deve ser realizado a cada três (3) anos, após dois (2) resultados negativos com intervalo de um (1) ano. Esta recomendação, denota a importância da realização do exame preventivo junto às mulheres que estejam em liberdade ou encarceradas.

No presente estudo foi possível identificar três (3) mulheres com lesão intraepitelial de baixo grau - LSIL (5,5\%) e uma (1) com lesão intraepitelial de algo grau - HSIL $(1,8 \%)$, percentual menor do que o encontrado em um estudo realizado com 233 mulheres em liberdade e com 190 mulheres reclusas em que foi identificada a ocorrência de 46 casos $(20,2 \%)$ de lesão intraepitelial de baixo grau e quatro (4) casos de lesão intraepitelial de alto grau (1, 2\%) (Plácido, 2012). Da mesma forma, pesquisa sobre "perfil sociodemográfico e achados citológicos no colo do útero no Estado de São Paulo", com amostra de 1.767 privadas de liberdade, demonstrou que 47 (2,66\%) casos foram de LSIL e 29 (1,64\%) de HSIL (Silveira, 2016).

De 55 mulheres que aceitaram realizar o PCCU, 21 (36,8\%) tiveram resultado sugestivo para Gardnerella vaginalis e $4(7,0 \%)$ apresentaram resultado sugestivo para HPV. A vulnerabilidade das mulheres no sistema prisional às IST é evidenciada na identificação de IST, tendo sido referido por mais de $25 \%$ delas, a sífilis, sendo comprovada pelo resultado reagente ao Teste Rápido em 14 mulheres. Estas realizaram o VDRL quantitativo para identificar se a infecção estava ativa ou se tratava de cicatriz sorológica, resultando em $(4: 28,6 \%)$ resultados reagentes, sendo estas, tratadas conforme protocolo do Ministério da Saúde para tratamento de sífilis. Este resultado corrobora a pesquisa realizada com mulheres privadas de liberdade, em que $2(5,0 \%)$ tiveram sífilis (Silva \& Ribeiro, 2013) e divergem de achados que mostraram que $14 \%$ das mulheres presas apresentaram maior prevalência para infecção por Trichomonas vaginalis (Nijhawan et al., 2012).

\section{Conclusão}

A primeira conclusão é que as mulheres privadas de liberdade são vulneráveis às IST. O estudo, ao se propor identificar IST em mulheres no sistema prisional de Santarém, Pará, mostrou o quão essencial é a atenção em saúde à população privada de liberdade, devendo ser pautada nos princípios da Política Nacional de Atenção Integral à Saúde da População Privada de Liberdade no Sistema Prisional (PNAISP), de forma qualificada em conjunção aos princípios e diretrizes do SUS, no âmbito da promoção, de prevenção e de recuperação da saúde.

Durante a realização do estudo, foi perceptível o quão distante o sistema prisional brasileiro, está do ideal humanitário, e quão urgente, radical e necessária são as mudanças a curto, médio e longo prazo, pois, dia a dia, as condições insalubres e até sub-humanas, diminuem e depreciam as poucas, porém, ainda existentes, chances de ressocialização.

Faz-se necessário reconhecer as mulheres em privação de liberdade como capazes de tomarem decisões sobre seus corpos e sua saúde. E para tal, é fundamental que tenham condições dignas e oportunidades de promoção e educação em saúde, bem como atendimento dentro dos princípios garantidos pelas políticas públicas, de acessibilidade, integralidade, resolubilidade e humanização da assistência em saúde. 
Ressalta-se que o presente estudo não tem a pretensão de esgotar as perspectivas de pesquisa acerca da temática, dessa forma, recomenda-se o desenvolvimento de pesquisas futuras que possam oferecer subsídios para o fortalecimento do conhecimento científico, e ofertar resultados mais concisos da prática realizada nos serviços de saúde, visando a atenção aos cuidados envolvendo prevenção, diagnóstico e tratamento das IST/HIV/aids no sistema prisional.

\section{Referências}

Ayres, J. R. C. M., Freitas, A. C., Santos, M. A. S., Saletti Filho, H. C., França Júnior, I. (2003). Adolescência e Aids: avaliação de uma experiência de educação preventiva entre pares. Interface (Botucatu). 7(12):113-28.

Barbosa, M. L., Silva, P. B. A., Cardins, K. K. B., Amaral, N. O., Costa, G. M. C. (2017). Assistência à saúde nas prisões brasileiras: uma revisão integrativa. $3^{\circ}$ Seminário Internacional de Pesquisa em Prisão. Recife - PE. UFPE. GT 13 - Saúde nas Prisões.

Belda Jr, W., Shiratsu, R., Pinto, V. V. (2009). Abordagem nas doenças sexualmente transmissíveis. Revista Brasileira de Dermatologia. 84(2): 151-59.

Boni, R., Veloso, V. G. E., Grinsztejn, B. (2014). Epidemiology of HIV in Latin America and the Caribbean. Curr. Opin. HIV AIDS. 2;9(2):192-8.

Brasil (2018). Infopen - Levantamento Nacional de Informações Penitenciárias - Infopen mulheres. (2 ${ }^{a}$ edição). Ministério da Justiça e da Segurança Pública. Distrito Federal - DF.

Buchalla, C. M., Paiva, V. (2002). Da compreensão da vulnerabilidade social ao enfoque multidisciplinar. Rev. Saúde Pública. 36 (4 Supl):117-9.

Dallabetta, G., Laga, M., \& Lamptey, P. (1997). Controle de Doenças Sexualmente Transmissíveis: Manual de Planejamento e Coordenação de Programas: Te Corá Editora / Associação Saúde da Família.

IBGE (2015) - Instituto Brasileiro de Geografia e Estatística.

Martins, T. A., Kerr, L. R. F. S., Kendall, C., Mota, R. M. S (2014). Cenário Epidemiológico da Infecção pelo HIV e AIDS no Mundo. Epidemiological setting of HIV infection and AIDS in the World. Rev Fisioter S Fun. 3(1):4-7.

Ministério da Saúde, Departamento de IST, AIDS e Hepatites Virais. Secretaria de Vigilância em Saúde. Protocolo clínico e diretrizes terapêuticas (PCDT): Atenção Integral as pessoas com Infecção Sexualmente Transmissíveis (IST).

Mome, R. K. B., Wiyeh, A. B., \& Kongnyuy, E. J. (2018). Eficácia do preservativo feminino na prevenção do HIV e infecções sexualmente transmissíveis: um protocolo de revisão sistemática. BMJ. 8: e023055. 10.1136/ bmjopen-2018-023055.

Naud, P. (1993). DST \&AIDS: Artes Médicas.

Neri, M. S., Oliveira, J. F., Nascimento, E. R., Gusmão, M. E. N., \& Moreira, V. S. (2011). Presas pelas drogas: características de saúde de presidiárias em Salvador, Bahia. Rev. Baiana de Enfermagem. 5(2) 121-132.

Nicolau, A. I. O., Ribeiro, S. G., Lessa, P. R. A., Monte, A. S., Bernardo, E. B. R., \& Pinheiro, A. K. B. (2012). Conhecimento, atitude e prática do uso de preservativos por presidiárias: prevenção das DST/HIV no cenário prisional. Rev. Esc. Enferm. USP. 46 (3):711-9.

Nijhawan, A. E., Chapin, K. C., Slloway, R., Andrea, S., Champion, J., \& Roberts, M. (2012). Prevalence and predictors of Trichomonas infection in newly incarcerated woman. Sex Transm. Dis. 39(12):1-12.

Organização Mundial da Saúde - OMS (2013). Infecções Sexualmente Transmissíveis (IST), a importância de um compromisso renovado com a prevenção e o controle das IST na obtenção de saúde sexual e reprodutiva global.

Organização Mundial da Saúde - OMS (2016). Estratégia global do setor de saúde sobre infecções sexualmente transmissíveis, 2016 -2021.

Organização Mundial da Saúde - OMS (2017). Infecções de transmissão sexual (ITS).

Organização Pan Americana de Saúde - OPAS (2017). Folha Informativa/HIV/aids.

Plácido, W. S. (2012). Epidemiologia da infecção pelo Papilomavírus Humano (HPV) em população feminina geral e população carcerária. Dissertação (Mestrado). Universidade Federal do Pará. Núcleo de Medicina Tropical. Belém, Pará.

Pereira, A. S., Shitsuka., D. M., Pereira, F. J., \& Shitsuka, R. (2018). Metodologia da pesquisa científica: UFSM, NTE.

Reis, C. B., \& Bernardes, E. B. (2011). O que acontece atrás das grades: estratégias de prevenção desenvolvidas nas delegacias civis contra HIV/AIDS e outras doenças sexualmente transmissíveis. Ciência \& Saúde Coletiva, 16(7):3331-3338.

Rufino, E. C., Andrade, S. S. C., Leadebal, O. D. C. P., Brito, K. K. G., Silva, F. M. C., Santos, S. H. (2016). Conhecimento de mulheres sobre IST/aids: intervindo com educação em saúde. Rev. Cienc. Cuid. Saúde. 15(1):9-16.

Silva, E. F., \& Ribeiro, E. R. (2013). Atenção à saúde da mulher em situação prisional. Revista Saúde e Desenvolvimento. 4(2).

Silveira, S. F. S. (2016). Perfil sociodemográfico e achados citopatológicos no colo do útero da população feminina privada de liberdade do Estado de São Paulo. Dissertação (Mestrado). Fundação Pio XII. Hospital do Câncer de Barretos. Barretos. São Paulo. 
Research, Society and Development, v. 10, n. 1, e49410112044, 2021 (CC BY 4.0) | ISSN 2525-3409 | DOI: http://dx.doi.org/10.33448/rsd-v10i1.12044

UNAIDS (2013). A ONU e a resposta à AIDS no Brasil: UNAIDS do Brasil.

UNAIDS (2013). Global Report: Unaids Report on the Global AIDS Epidemic 2013 :148 p.

UNAIDS (2015). Chegando a zero. Estratégia do UNAIDS/ONUSIDA. 2011- 2015. Brasília-DF, Brasil.

World Health Organization - WHO (2103). Sexually Transmitted Infections (STIs), The importance of a renewed commitment to STI prevention and control in achieving global sexual and reproductive health.

Zaninelli, G. (2015). Mulheres encarceradas: Dignidade da pessoa humana, gênero, legislação e políticas públicas. 2015. Dissertação (Mestrado). Universidade Estadual do Norte do Paraná. Programa de Mestrado em Ciências Jurídicas. Jacarezinho - Paraná. 